\title{
High-Altitude Medicine: Important for Trekkers and Mountaineers, Essential for Progress in Medicine
}

\author{
Yves Allemann ${ }^{\mathrm{a}, *}$, Urs Scherrer ${ }^{\mathrm{b}}$ \\ ${ }^{a}$ Cardiology, Swiss Cardiovascular Center Bern, University Hospital, Bern, Switzerland \\ ${ }^{\mathrm{b}}$ Department of Internal Medicine, Centre Hospitalier Universitaire Vaudois, Lausanne, Switzerland
}

It is a pleasure to dedicate an issue of Progress in Cardiovascular Diseases to high-altitude medicine, and we gratefully thank the editors for giving us this opportunity.

The readers will discover that the frontiers of highaltitude medicine are almost infinite and often extend to low altitude. Why is this so? Progress in medicine is intimately linked with the understanding of underlying mechanisms of disease. At high altitude, the ambient hypoxia triggers a series of physiologic responses that affect principally the respiratory, cardiovascular, neuroendocrine, and renal systems with the goal of maintaining adequate cellular oxygenation. At low altitude, the same systems may be activated with the same goal. The trigger, however, is not ambient hypoxia but hypoxemia that is the consequence of an organ insufficiency, usually the heart or the lung. Therefore, the ultimate goal of most high-altitude researchers is not only to understand physiologic (mal)adaptation to hypoxia for the benefit of the millions exposing themselves to high altitude, but to think beyond that, imagining how the knowledge gained from field research at high altitude may be applied to the much larger number of patients with hypoxia/ hypoxemia-associated diseases.

A recent example of research from our own group may illustrate this state of mind. As detailed elsewhere in this issue, high-altitude pulmonary edema (HAPE) offers a unique model to study underlying mechanisms of pulmonary edema in humans (see p 485 by Scherrer et al).

Statement of conflict of interest: See page 450.

* Address reprint requests to Yves Allemann, MD, FESC, Cardiology, Swiss Cardiovascular Center Bern, University Hospital, CH-3010 Bern, Switzerland.

E-mail address: yves.allemann@insel.ch (Y. Allemann).
Exaggerated hypoxic pulmonary hypertension is a hallmark of HAPE, and its triggering of alveolar fluid flooding was thought to represent the main pathogenic mechanism. Epidemiologic studies suggest that pathologic events during the fetal/perinatal period may lead to systemic vascular dysfunction later in life. In a study on fetal/ perinatal programming of pulmonary vascular dysfunction performed at $4559 \mathrm{~m}$, we found that healthy adults who had transient lack of oxygen during the first days after birth display exaggerated hypoxic pulmonary hypertension, similar in magnitude to that observed in HAPE-prone subjects. ${ }^{1}$ Surprisingly, however, none of these young individuals developed $\mathrm{HAPE},{ }^{2}$ indicating that exaggerated pulmonary hypertension per se is not always sufficient to trigger HAPE and that additional mechanisms play a role. In subsequent studies, we showed that impaired alveolar fluid clearance related to a defect of the transepithelial sodium transport represents such an additional mechanism because stimulation of this transport with a $\beta$-adrenergic agonist prevents lung edema in HAPE-prone subjects. ${ }^{3}$ This high-altitude study demonstrated for the first time that sodium-driven alveolar fluid clearance plays a pathogenic role in pulmonary edema in humans and may, therefore, represent an appropriate new target for its therapy. Subsequent clinical studies then demonstrated that $\beta$-adrenergic stimulation of this transport decreases extravascular lung water in patients with acute lung injury ${ }^{4}$ and accelerates the resolution of pulmonary edema after lung resection. ${ }^{5}$ Other examples on how high altitude may represent a natural research laboratory for the study of cardiovascular physiology and pathophysiology are presented by Scherrer et al (p 451).

As stated above, the primary goal of the physiologic adaptation of the cardiovascular system and the lungs to acute high-altitude exposure is to ensure maximal 
Abbreviation and Acronym

HAPE = high-altitude pulmonary edema oxygen transport to the organ tissues despite the ambient lack of oxygen. These different, early and late, cardiovascular responses to hypoxia, as well as their limitations, are reviewed in detail by Naeije ( $p$ 456).

There are 3 syndromes of maladaptation to acute highaltitude exposure, also called high-altitude illnesses: acute mountain sickness, high-altitude cerebral edema, and HAPE. The pathophysiology, prevention, and treatment of the very frequent but usually benign syndrome of acute mountain sickness and the fortunately much less frequent high-altitude cerebral edema are summarized in a comprehensive review by Imray and colleagues ( $p$ 467).

The new insight in the pathogenesis of HAPE provided by Scherrer et al ( $p$ 485) is very exciting and, in many ways, offers new ideas and concepts that could be tested and translated into clinical medicine.

The in-depth review of Sartori et al (p 493) complements the previous article by focusing on new physiologic and pathologic concepts of lung fluid movements under hypoxic conditions and their potential role in clinical practice.

High-altitude pulmonary edema is a life-threatening problem, and physicians need to know how to advise individuals planning high-altitude activities. The article by Maggiorini et al ( $\mathrm{p}$ 500) provides this up-to-date information on how to treat and prevent this important disease.

Because of the development of modern transportation systems, ascent to high altitude has become very easy. Therefore, not only mountaineers, but ever-increasing numbers of patients with cardiovascular disease are exposing themselves to high altitude; and counseling of such patients has become very important. The extent of the risk is not easily quantifiable and depends largely on the altitude reached, the acclimatization status, and the leisure time activity planned. Burtscher and Ponchia provide up-to-date information regarding risk stratification in cardiovascular patients ( $p$ 507). In a very practically oriented companion article, Rimoldi et al provide recommendations on how to assess and advise cardiovascular patients planning high-altitude exposure and activity (p 512).

Sometimes, a hypoxic environment is deliberately sought. This is the case for endurance athletes who try to naturally augment their oxygen transport capacity. Several approaches on how to achieve this goal efficiently have been advocated and debated. Should the athlete "live high and train low" or rather "live low and train high," or is that all old-fashioned? Vogt and Hoppeler bring together the latest concepts on that topic (p 525).

High-altitude hypoxia is sometimes deliberately sought and sometimes just the destiny, as for the dwellers permanently living at high altitude in the Andes, in the Himalayas, or in other mountainous regions around the globe. What are the cardiovascular adaptation mechanisms that have been developed by these high-altitude populations, particularly by the Bolivian Aymaras? How do they differ from chronic adaptation mechanisms of white people living at the same altitude? These (dis)similarities and their possible positive or negative long-term consequences on cardiopulmonary health are discussed by Stuber et al (p 534).

Adaptation mechanisms to hypoxia can sometimes go beyond their primary goal of maintaining adequate tissue oxygenation. This is the case in chronic mountain sickness, a major public health problem. Affected patients develop, usually insidiously over time, excessive erythrocytosis, hypoxemia, and pulmonary hypertension that are associated with a corollary of nonspecific symptoms having a major negative impact on quality of life. The cardiovascular consequences of chronic mountain sickness are brightly explained by León-Velarde et al in the last article of this special issue (p 540).

\section{Statement of Conflict of Interest}

Both authors declare that there are no conflicts of interest.

\section{References}

1. Sartori C, Allemann Y, Trueb L, et al: Augmented vasoreactivity in adult life associated with perinatal vascular insult. Lancet 1999;353: 2205-2207.

2. Sartori C, Allemann Y, Trueb L, et al: Exaggerated pulmonary hypertension is not sufficient to trigger high-altitude pulmonary oedema in humans. Schweiz Med Wochenschr 2000;130:385-389.

3. Sartori C, Allemann Y, Duplain H, et al: Salmeterol for the prevention of high-altitude pulmonary edema. N Engl J Med 2002;346: 1631-1636.

4. Perkins GD, McAuley DF, Thickett DR, et al: The beta-agonist lung injury trial (BALTI): a randomized placebo-controlled clinical trial. Am J Respir Crit Care Med 2006;173:281-287.

5. Licker M, Tschopp JM, Robert J, et al: Aerosolized salbutamol accelerates the resolution of pulmonary edema after lung resection. Chest 2008;133:845-852. 